\title{
EGFR and NF-kB: partners in cancer
}

\author{
Kateryna Shostak ${ }^{1,2,3}$ and Alain Chariot $^{1,2,3,4}$ \\ ${ }^{1}$ Interdisciplinary Cluster for Applied Genoproteomics (GIGA), Centre Hospitalier Universitaire (CHU), Sart-Tilman, Liege, Belgium \\ ${ }^{2}$ Laboratory of Medical Chemistry, University of Liege, CHU, Sart-Tilman, Liege, Belgium \\ ${ }^{3}$ GIGA-Signal Transduction, University of Liege, CHU, Sart-Tilman, Liege, Belgium \\ ${ }^{4}$ Walloon Excellence in Life Sciences and Biotechnology (WELBIO), Wavre, Belgium
}

\begin{abstract}
Oncogenic proteins cooperate to promote tumor development and progression by sustaining cell proliferation, survival and invasiveness. Constitutive epidermal growth factor receptor (EGFR) and nuclear factor $\kappa b$ (NF-kB) activities are seen in multiple solid tumors and combine to provide oncogenic signals to cancer cells. Understanding how these oncogenic pathways are connected is crucial, given their role in intrinsic or acquired resistance to targeted anticancer therapies. We review molecular mechanisms by which both EGFR- and NF-kB-dependent pathways establish positive loops to increase their oncogenic potential. We also describe how NF-KB promotes resistance to EGFR inhibitors.
\end{abstract}

\section{Constitutive EGFR signaling in solid tumors}

Oncogenic proteins (see Glossary) cooperate to efficiently drive tumor development and progression. Cancer cells are indeed characterized by a powerful signaling network showing multiple connections to survive, proliferate and to resist to targeted anticancer therapies. Constitutive signaling from the EGF receptor (EGFR/HER1/ERBB1), a protein of $170 \mathrm{kDa}$ and a member of the ERBB family of receptor tyrosine kinases (RTKs; Box 1), crucially promotes cell survival, proliferation, and invasiveness [1]. A variety of EGF peptides trigger EGFR dimerization and phosphorylation of multiple tyrosine residues in its cytoplasmic tail. Those phosphorylated EGFR residues provide docking sites for cytoplasmic SRC homology 2 (SH2) and phosphotyrosine-binding (PTB) domain-containing proteins to specifically trigger PKC, PI3K/AKT/mTOR, SRC, STAT and RAS/RAF/MEK1/ERK1/2 activation (Figure 1) [2,3].

More than 100 EGFR-interacting proteins have been described so far [4]. Among them is growth factor receptorbound protein 2 (GRB2) which binds to phosphorylated tyrosines 1068, 1086, and 1148. RAS is subsequently activated by phosphorylation, a modification that relies on son of sevenless (SOS). Activated RAS binds to RAF, and this interaction leads to mitogen-activated protein kinase kinase 1 (MEK1) followed by extracellular signalregulated kinases 1/2 (ERK1/2) phosphorylations [5,6]. RAS activation also relies on the recruitment of the SRC homology domain-containing adaptor protein $\mathrm{C}$ (SHC) to phosphorylated EGFR [7]. The p85 regulatory

Corresponding author: Chariot, A. (Alain.chariot@ulg.ac.be).

Keywords: EGFR; gefitinib; erlotinib; NF-кB; resistance; tumor-initiating cells.

$1471-4914 /$

(C) 2015 Elsevier Ltd. All rights reserved. http://dx.doi.org/10.1016/j.molmed.2015.04.001 subunit of phosphatidylinositol-3-kinase (PI3K), the kinase SRC, and protein tyrosine phosphatases such as PTP1B, SHP1, and SHP2 also associate with distinct phosphorylated EGFR residues (Figure 1) [8]. EGFR directly or indirectly (through JAK) activates signal transducer and activator of transcription (STAT) members. EGFR phosphorylation also triggers STAT activation through SRC as well as the activation of PI3K that subsequently promotes AKT activation. Activated AKT targets multiple substrates, including mammalian target of rapamycin (mTOR). Phosphoinositide-specific phospholipase C $\gamma 1$ (PLC $\gamma 1$ ) binds to EGFR through its SH2 domain, becoming activated and hydrolyzing phosphatidylinositol 4,5-bisphosphate to diacylglycerol (DAG) and inositol trisphosphate (IP3). DAG then triggers the activation of serine/threonine kinase protein kinase $\mathrm{C}$ (PKC) (Figure 1). $\mathrm{NF}-\kappa \mathrm{B}$ is also activated through the IKK complex upon EGFR phosphorylation.

Overexpression and activating mutations of EGFR, which have been reported in up to $30 \%$ of solid tumors (including breast, colorectal, lung, pancreatic, gastric, head and neck cancer, and glioblastomas), generally correlate with a poor prognosis [9]. A variety of solid tumors, including lung carcinomas, are indeed dependent upon EGFR activation, and this makes them sensitive to EGFR inhibitors such as erlotinib or gefitinib (see [10] for a full description of EGFR inhibitors) [11]. Some patients suffering from lung cancer are highly responsive to gefitinib because of activating EGFR point mutations or in-frame deletions (Figure 1) $[12,13]$. These genetic alterations

\section{Glossary}

Erlotinib: a pharmacological inhibitor that binds in a reversible fashion to the ATP binding site of the EGFR receptor. This EGFR inhibitor showed a survival benefit in the treatment of lung cancer in Phase III clinical trials. Erlotinib is more effective in patients with EGFR activating mutations.

Gefitinib: the first EGFR inhibitor approved for the treatment of non-small cell lung carcinoma. Similarly to erlotinib, this drug binds in a reversible fashion to the ATP-binding site of the EGFR receptor.

Oncogenes: gene candidates coding for proteins involved in tumor development. Many oncogenes are amplified or targeted by activating mutations to act in a genetically dominant manner.

Paronychia: a bacterial or fungal infection of the hand or foot where the nail and skin meet.

Polyubiquitination: a post-translational modification in which several copies of $7 \mathrm{kDa}$ ubiquitin are bound to a protein substrate to create a polyubiquitin chain. This covalent modification involves three sequential enzymatic reactions catalyzed by the E1 (ubiquitin-activating), E2 (ubiquitin-conjugating), and E3 (ubiquitin ligase) enzymes [78].

Xerosis: a skin disease involving the integumentary system. Symptoms include the peeling of the outer skin layer, itching, and skin cracking. 


\section{Box 1. ERBB members}

The ERBB receptors include the EGF receptor (EGFR, also named HER1), ERBB2 (HER2/Neu), ERBB3 (HER3), and ERBB4 (HER4), and belong to the family of type I receptor tyrosine kinases (RTK) [79]. ERBB receptors are mainly expressed in epithelial, mesenchymal, and neuronal cells, as well as in their progenitors. The receptors share an extracellular ligand-binding domain, a single membranespanning region, and a cytoplasmic domain that includes a juxtamembrane domain, a region harboring an intrinsic tyrosine kinase activity, as well as a C-terminal domain [80]. ERBB receptors are bound by EGF-family peptides. These ligands include EGF, transforming growth factor (TGF)- $\alpha$, amphiregulin (AR), and epigen (EPG) which bind to EGFR; $\beta$-cellulin (BTC), heparin-binding EGF (HB-EGF), and epiregulin (EPR) which bind to both EGFR and ERBB4; and neuregulins (NRGs) such as NRG-1 and NRG-2 that are known to bind to both ERBB3 and ERBB4, as well as NRG-3 and NRG-4 acting as ligands for ERBB4 only $[79,80]$. NRG-1 has several isoforms (type I NRG-1, also named 'heregulin', to type VI NRG-1). ERBB2 does not directly bind to any of these peptides, whereas ERBB3 is devoid of any strong kinase activity and only signals when bound to other ERBB members.

target the cytoplasmic domain of EGFR in lung adenocarcinomas, while, in contrast, mutations in glioblastomas showing constitutive EGFR signaling target the extracellular domain of this receptor [14,15]. Exons 18-21 of the tyrosine kinase domain of EGFR harbor all key mutations. About $40 \%$ of genetic alterations found in highly-responsive patients are in exon 21 , and the most common is L858R. Some in-frame deletions in exon 19 $(\triangle \mathrm{E} 746-\mathrm{A} 750$ as well as other deletions in this exon) account for about $46 \%$ of the reported EGFR genetic alterations found in highly-responsive patients. Point mutations in exon 18 (G719A, G719S, and G719C) have been described in about $1 \%$ of those patients. Less-frequent EGFR mutations underlying drug sensitivity or resistance have been described elsewhere [16,17].

The therapeutic effectiveness of EGFR inhibitors has been disappointing due to the emergence of resistant cancer cells. Virtually all patients who initially respond to EGFR inhibitors become resistant to these drugs as a result of acquired EGFR mutations [18]. The most clinically relevant EGFR mutation found in 50\% of the cases showing acquired resistance to EGFR inhibitors (gefitinib and erlotinib) is the T790M mutation located in exon 20 (Figure 1) [19]. This mutation, which is located within the ATP-binding site of the kinase domain, causes steric hindrance for access of the inhibitor to the cleft owing to the bulkiness of the methionine sidechain [20]. The use of irreversible inhibitors of the EGFR kinase activity to treat patients harboring this mutation is an attractive therapeutic approach, and has prompted the search for new EGFR inhibitors that specifically target the EGFR T790M mutation. Several molecules have been identified that are more specific for this mutated EGFR than for the wild type receptor [21].

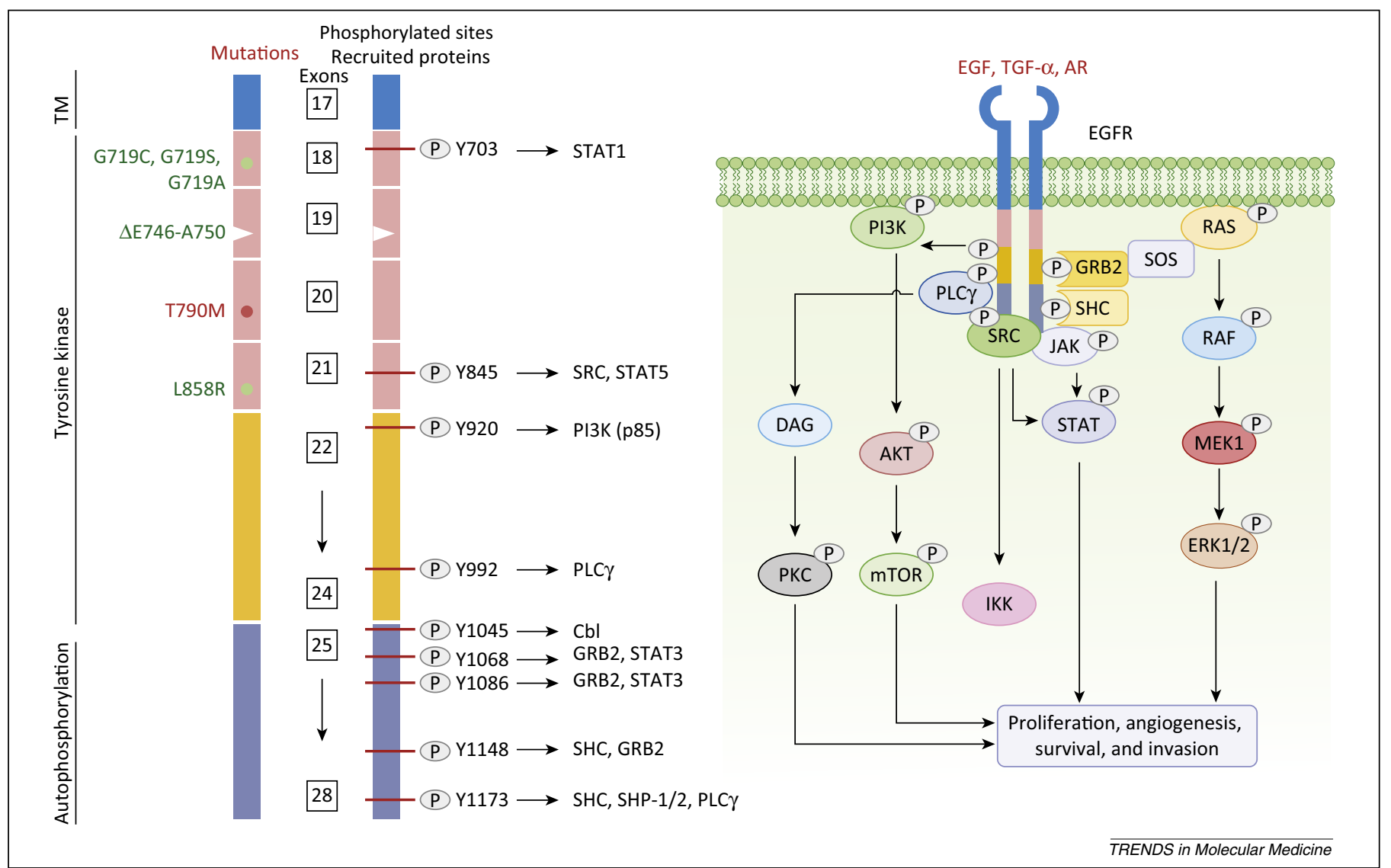

Figure 1. EGFR-dependent signaling pathways and EGFR mutations in solid tumors. Ligands of EGFR homodimers include EGF, TGF- $\alpha$, and AR. Examples of proteins recruited on tyrosine-phosphorylated EGFR residues are listed on the left and the most characterized signaling pathways activated upon EGFR phosphorylation are illustrated on the right. The most frequent mutations linked to drug sensitivity and found in drug responders are depicted in green. The most clinically relevant EGFR mutation (T790M) that promotes resistance to EGFR inhibitors is represented in red. Abbreviations: AR, amphiregulin; EGF, epidermal growth factor; EGFR, epidermal growth factor receptor; $\mathrm{P}$, phosphorylation; TGF- $\alpha$, transforming growth factor $\alpha$; TM, transmembrane. 
EGFR point mutations are not the only mechanism by which cancer cells are (or become) resistant to EGFR inhibitors. Activation of other RTKs such as ERBB2/ HER2 also occurs in cells resistant to cetuximab, an EGFR-targeting monoclonal antibody, which paves the way for the dual inhibition of both EGFR and HER2 to improve the clinical response [22]. Signaling from ERBB3/ HER3 is also specifically activated in epithelial malignancies treated with EGFR inhibitors [23-25]. Although HER3 lacks intrinsic kinase activity, it nevertheless strongly activates AKT signaling as a dimer with HER2 [26,27]. Therefore, a variety of pharmacological approaches, including HER3-blocking antibodies, have been recently developed to circumvent resistance $[10,28]$. It is currently unclear whether the use of multiple ERBB inhibitors is the best approach, or whether other types of inhibitors have to be combined with them. In any case, dissecting all relevant oncogenic pathways is of paramount importance to identify new mechanisms underlying resistance to EGFR inhibitors, and to define the best combination of specific drugs to fight epithelial malignancies. This review will focus on molecular mechanisms by which the transcription factor $\mathrm{NF}-\kappa \mathrm{B}$ is activated upon EGFR activation, as well as on $\mathrm{NF}-\kappa \mathrm{B}-$ dependent pathways underlying resistance to EGFR inhibitors.

\section{Molecular mechanisms linking EGFR signaling to NF-кB activation}

Growth factors promote NF-кB activation through ERBB members, but the underlying mechanisms are only now starting to be elucidated. The family of NF- $\mathrm{KB}$ transcription factors are typically activated by proinflammatory cytokines such as tumor necrosis factor (TNF)- $\alpha$ or IL$1 \beta$, as well as by Toll-like receptor (TLR) ligands through extremely well defined signaling cascades (Box 2) [29]. Early studies demonstrated that EGF triggers NF-кB activation through the proteasome-mediated degradation of the inhibitory molecule I $\kappa \mathrm{B} \alpha$ in estrogen receptor $\mathrm{ER} \alpha-$ negative breast cancer cells and in lung cancer-derived cells $[30,31]$. Heregulin also triggers $\mathbf{N F}-\kappa \mathrm{B}$ activation through the IKK complex in ER $\alpha$-negative and ERBB2positive breast cancer cells [32]. In addition, constitutive EGFR signaling leads to NF-кB activation through IкB $\alpha$ phosphorylation on serines 32 and 36 in prostate cancer cells [33].

Although it is now well established that EGF activates NF-кB through the IKK complex (that includes both catalytic subunits IKK $\alpha$ and IKK $\beta$ as well as the scaffold protein NEMO/IKK $\gamma$ ), signaling molecules that link EGFR activation to the IKK complex have only been recently characterized (Figure 2). Distinct pathways have been elucidated in detail, but it remains unclear whether they are activated simultaneously or in a cell specific manner. EGF stimulation in prostate and breast cancer cells, as well as in EGFR-overexpressing glioblastoma-derived cells, triggers $\mathrm{PKC} \varepsilon$ monoubiquitination at Lys 321 in a PLC $\gamma 1$-dependent manner [34]. PKC $\varepsilon$ monoubiquitination relies on the E3 ligase RINCK1, but not on the linear ubiquitin assembly complex (LUBAC) that includes HOIL-1L and HOIP. Monoubiquitinated PKCe recruits the IKK complex to the plasma domain through a physical

\section{Box 2. The NF-kB family of transcription factors}

NF-kB proteins, which include RelA (also named p65), RelB, and cRel, share a N-terminal Rel homology domain (RHD) that is required for homo- and heterodimerization and for binding to sequencespecific DNA-binding sites in the promoters of $\sim 200$ target genes. These NF-кB proteins harbor a C-terminal transactivating domain (TAD). NF-kB proteins also include $\mathrm{p} 50$ and p52, which are

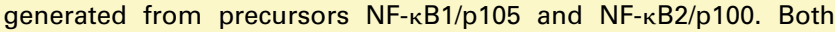
p50 and p52 lack any TAD and therefore rely on other members to drive gene expression of NF-кB-target genes. In unstimulated cells, $\mathrm{NF}-\mathrm{kB}$ proteins are sequestered in the cytoplasm through binding to inhibitory molecules whose prototype is $I_{\kappa} B \alpha$ [81]. Other inhibitory

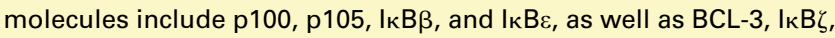

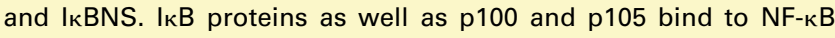
dimers through multiple ankyrin repeats. Stimulation with a variety of stimuli, such as proinflammatory cytokines (e.g., TNF $\alpha$ and IL-1 $\beta$ ), Toll-like receptor ligands [e.g., lipopolysaccharide (LPS) and doublestranded RNA (dsRNA)], triggers NF-кB activation through the socalled 'classical' or 'canonical' pathway. This signaling pathway leads to the IKK complex, composed of both kinases IKK $\alpha$ and IKK $\beta$ assembled by the scaffold protein NEMO/IKK $\gamma$. The IKK complex phosphorylates $\mathrm{I}_{\kappa} \mathrm{B} \alpha$ on $\mathrm{N}$-terminal serines, and this triggers its degradative polyubiquitination through the proteasome. NF-кB dimers (mainly p50/p65 and p50/c-Rel) are consequently released and translocated to the nucleus to drive gene transcription of candidates involved in innate immunity, inflammation, proliferation, and survival. Growth factors can also trigger the activation of the IKK complex through signaling pathways described in Figure 2 in main text.

The 'alternative' or 'non-classical' NF-кB-activating pathway is triggered by cytokines such as BAFF and lymphotoxin- $\beta$, and leads to the activation of an IKK $\alpha$ homodimer, which phosphorylates p100. This inhibitory molecule is subsequently processed to generate $\mathrm{p52}$. NF-kB dimers ( $552 /$ RelB) move into the nucleus to drive the expression of candidates involved in adaptive immunity, as well as in lymphoid organogenesis. The activation of all NF-кB signaling pathways relies on the sequential phosphorylation of multiple proteins, as well as on the polyubiquitination of key actors through several types of chains, the most characterized being the K48-linked chain, which triggers the degradation of its substrate or both linear and K63-linked chains, which enhance protein-protein interactions [82].

interaction with a ubiquitin-binding domain in the zinc finger of $\mathrm{NEMO} / \mathrm{IKK} \gamma$. PKC $\varepsilon$ then activates $\mathrm{NF}-\kappa \mathrm{B}$ through IKK $\beta$ phosphorylation at Ser 177. This pathway ultimately drives tumor growth by inducing the expression of pyruvate kinase 2 (PKM2), the enzyme involved in the rate-limiting final step of glycolysis. The IKK $\beta$-phosphorylating kinase TGF $\beta$-activating kinase 1 (TAK1) appears to be dispensable for this pathway, meaning that EGF as well as proinflammatory cytokines such as TNF $\alpha$ and IL$1 \beta$ activate $\mathrm{NF}-\kappa \mathrm{B}$ through distinct signaling pathways that will nevertheless converge at the IKK complex [34].

EGF-dependent NF- $\mathrm{KB}$ activation in some breast and lung cancer-derived cells also relies on the scaffold protein caspase recruitment domain (CARD), membrane-associated guanylate kinase-like domain protein 3 (CARMA3; also referred to as CARD10), and B cell lymphoma protein 10 (BCL-10) [35]. Interestingly, both CARMA3 and BCL-10 also promote GPCR- and PKC-dependent NF- $\mathrm{KB}$ activation when complexed with mucosa-associated lymphoid tissue lymphoma translocation gene 1 (MALT1), but it is currently unclear whether MALT1 is actually required in EGF-dependent IKK phosphorylation [36,37]. MALT1, as a subunit of the CBM (CARD10-BCL-10-MALT1) complex, recruits the E3 ligase TRAF6, which forms K63-linked polyubiquitin 


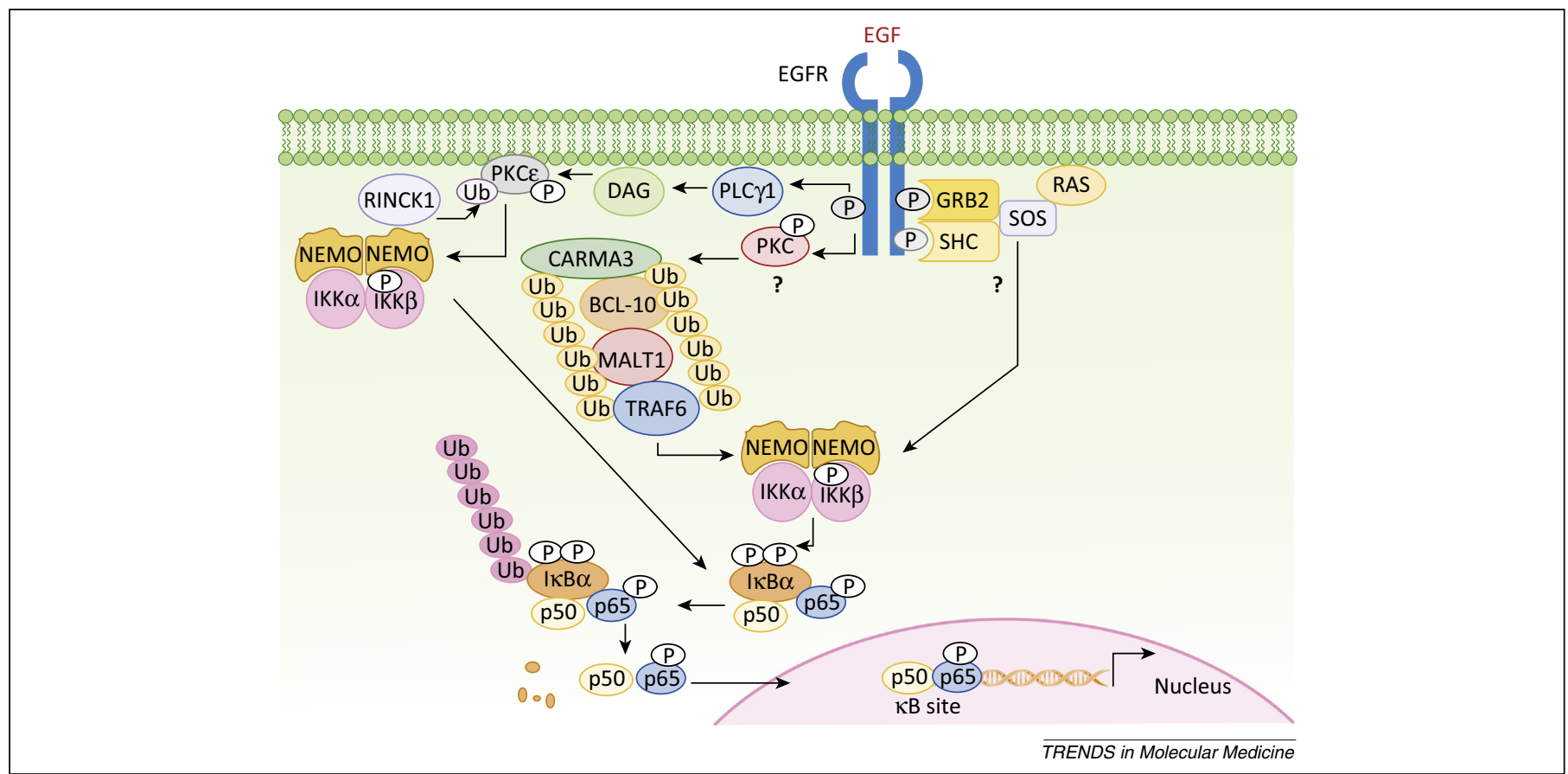

Figure 2. Molecular mechanisms by which EGFR activates NF-кB. EGF binding triggers EGFR phosphorylation and its association with the SOS-GRB2 protein complex. RAS is subsequently recruited and activated at the cell membrane to promote MEK1 and ERK1/2 activation (not depicted), a pathway that relies on the guanine nucleotide exchange catalytic activity of SOS. Constitutive EGFR-dependent NF-kB activation relies on SOS, which triggers IKK $\beta$ activation, a pathway that does not require the catalytic activity of SOS [39]. EGFR phosphorylation (P) also triggers PLC $\gamma 1$ activation, followed by DAG production and PKC $\varepsilon$ monoubiquitination (Ub) by the E3 ligase RING finger protein that interacts with $\mathrm{C}$ kinase 1 (RINCK1) bound to HOIL-1L and HOIP. Monoubiquitinated PKC $\varepsilon$ associates with a ubiquitin-binding domain within the zincfinger motif of NEMO to recruit the IKK complex to the plasma membrane. PKC $\varepsilon$ can then phosphorylate IKK $\beta$ to trigger NF- $\mathrm{B}$ activation [34]. EGF-dependent NF- $\mathrm{B}$ activation in cancer cells also relies on the CBM complex that includes CARMA3 and BCL-10. This complex also includes MALT1, but it is currently unclear whether this protein is required for NF-KB activation. The CBM complex activates IKK $\beta$ through TRAF6, an E3 ligase known to generate K63-linked polyubiquitin chains bound by NEMO. Signal transduction through the CBM complex relies on PKC activation, but the PKC isoform that triggers EGF-dependent IKK phosphorylation through this cascade has not

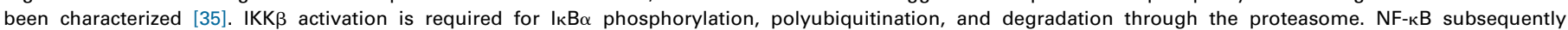
translocates to the nucleus to drive the expression of its target genes.

chains, to promote IKK activation through TAK1 in T lymphocytes [38]. Because MALT1 and BCL-10 are polyubiquitinated by TRAF6, they could be bound by NEMO/ IKK $\gamma$ in a TAK1-independent manner, a model that fits with the reported dispensable role of TAK1 in EGF-dependent IKK $\beta$ phosphorylation [34]. Activation of the CBM complex relies on PKC activation, but the PKC isoform that links EGFR activation to CARMA3 has not been identified. Whether PKC $\varepsilon$ fulfills this function remains to be demonstrated.

An additional pathway that links EGFR activation to $\mathrm{NF}-\kappa \mathrm{B}$ involves the guanine nucleotide exchange factor SOS1 [39]. Upon EGF stimulation, SOS1 binds to phosphorylated EGFR through the adaptor protein GRB2, which then triggers RAS activation at the plasma membrane [40]. Interestingly, its GDP-GTP exchange activity, known to be crucial for EGF-dependent MAP kinases activation, is dispensable for NF- $\mathrm{kB}$ activation upon EGF stimulation, and this supports the notion that SOS1 may also act as a scaffold protein to transmit oncogenic signals [39]. Nevertheless, signaling molecules that link SOS1 to the IKK complex are totally unknown (Figure 2).

These studies have convincingly demonstrated that growth factors promote NF-кB activation through signaling pathways whose initial steps are largely distinct from those triggered by proinflammatory cytokines. These signaling cascades are believed to crucially contribute to tumor development and progression through the expression of NF-кB-dependent genes that promote cell proliferation and survival.

\section{Crosstalk between EGFR- and NF-kB-dependent pathways through the transcriptional induction of target genes}

While growth factors trigger NF-кB-activating cascades upon binding to ERBB members, the transcriptional induction of some NF-кB target genes also feeds back to impact on EGFR-dependent signaling pathways. In this context, KIAA1199 is transcriptionally induced by NF- $\mathrm{kB}$ proteins in transformed keratinocytes as well as in breast cancerderived cells (Figure 3) [41,42]. The oncogenic human papillomavirus (HPV) also positively regulates KIAA1199 gene transcription through BCL-3 in cervical cancer cells [41]. KIAA1199 promotes EGFR stability by limiting its EGFdependent degradation in lysosomes, and therefore positively regulates EGFR signaling [41]. KIAA1199 actually limits semaphorin 3A-dependent cell death by promoting EGFR phosphorylation and as well as EGF-dependent epithelialmesenchymal transition (EMT) in cervical cancer cells [41]. As such, KIAA1199 links NF-кB-dependent gene transcription to EGFR signaling to sustain cell survival and invasion [41]. Another example of positive correlation between NF- $\mathrm{kB}$ and EGFR activities has been described in head and neck squamous cell carcinomas (HNSCCs) in which IKK $\alpha$ and/or $\beta$ knockdown significantly decreased 


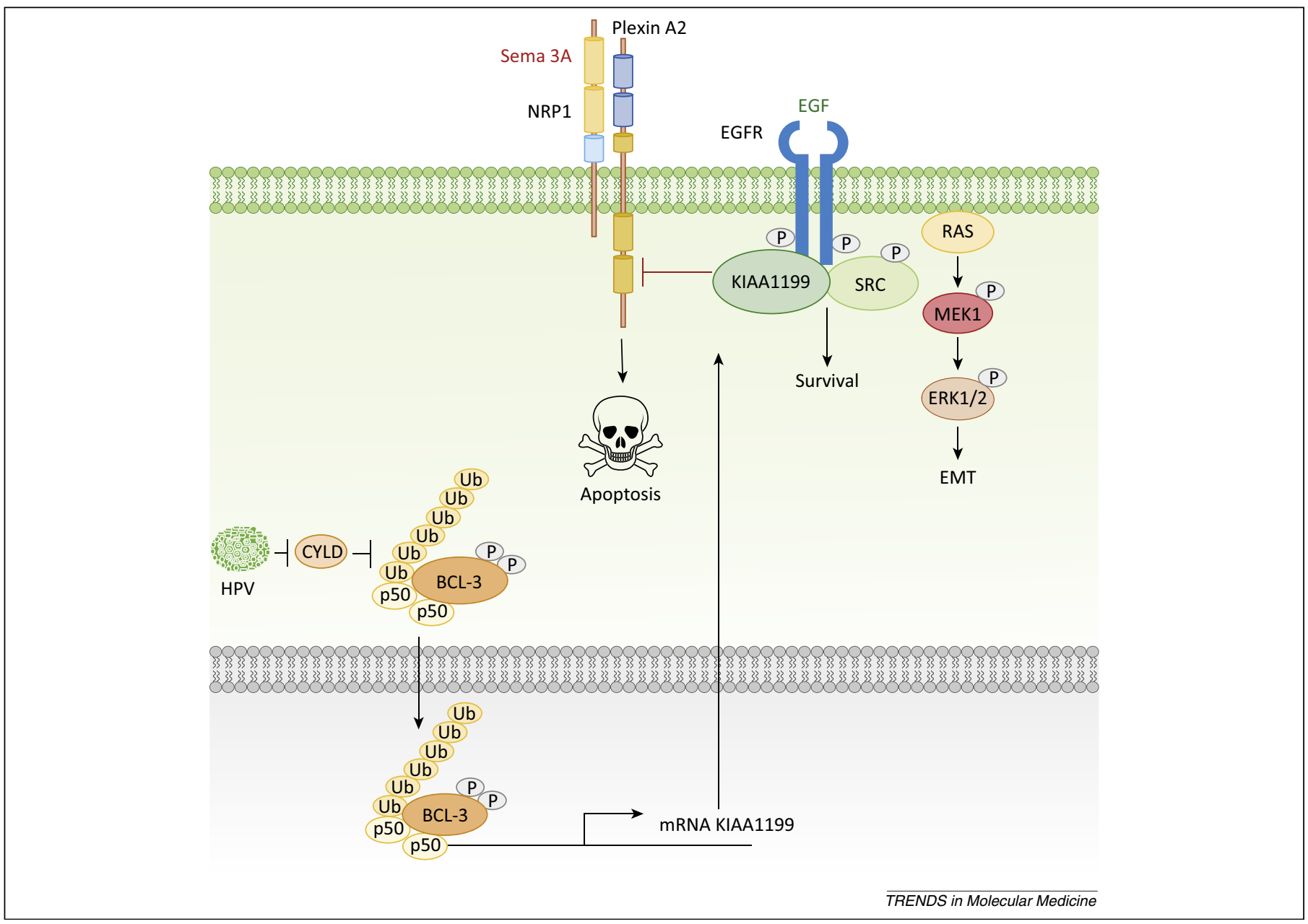

Figure 3. The NF-кB-induced protein KIAA1199 promotes EGFR stability and signaling and protects from semaphorin $3 A-m e d i a t e d$ cell apoptosis. Human papillomavirus (HPV) infection in keratinocytes inhibits CYLD, a ubiquitin C-terminal hydrolase. As a result, the non-degradative K63-linked polyubiquitination of BCL-3, a p50-binding protein, is enhanced, leading to its nuclear translocation. Nuclear BCL-3 drives KIAA1199 gene transcription. KIAA1199 binds to plexin A2 to limit semaphorin $3 A$-dependent cell death, and also stabilizes EGFR to promote EGF-dependent SRC and ERK1/2 activation, and subsequent epithelial-mesenchymal transition (EMT) [41].

EGFR mRNA and protein levels [43]. In contrast to this $\mathrm{NF}-\mathrm{\kappa B}$ signaling pathway that positively regulates EGFR signaling, EGFR expression is negatively regulated at the transcriptional level by receptor-interacting kinase (RIPK1), which is typically activated by proinflammatory and NF-кB-activating cytokines such as TNF $\alpha$ [44]. RIPK1 indeed appears to interfere with Sp1 activity, a transcription factor that promotes EGFR mRNA synthesis [44]. Therefore, multiple feedback loops involving the transcriptional induction of target genes that link both EGFR and NF-кB-dependent pathways have been described, even if they do not systematically lead to the establishment of positive loops.

\section{NF-кB activation as a mechanism for resistance to EGFR inhibitors}

NF- $\mathrm{kB}$-activating cascades promote resistance to chemotherapy through multiple mechanisms, including the transcriptional induction of multidrug resistance gene-1 (MDR1) in colon cancer cells [45]. Recent studies have also defined mechanisms by which resistance occurs through crosstalk between EGFR- and NF-кB-dependent pathways. The tyrosine kinase FER, known to be activated by EGFR and PDGFR upon ligand engagement, promotes resistance to quinacrine, a drug with antimalarial and anticancer effects, when overexpressed in prostate cancer cells [46-48]. Mechanistically, FER binds to EGFR to enhance its phosphorylation on tyrosine residues, which leads to NF-кB activation through an AKT-independent pathway [46].

An unbiased screen for oncogenic pathways underlying resistance to EGFR inhibitors led to the identification of multiple candidates involved in NF-кB signaling [49]. Indeed, an unbiased short hairpin RNA (shRNA)-based high-throughput screen carried out in lung cancer-derived H1650 cells insensitive to EGFR inhibitors led to the identification of several candidates, many of which act in $\mathrm{NF}-\kappa \mathrm{B}$-activating cascades [49]. Consistent with a role of $\mathrm{NF}-\kappa \mathrm{B}$ in the resistance to EGFR inhibitors, the genetic or pharmacologic inhibition of NF- $\mathrm{KB}$ increased the sensitivity to erlotinib in several models of EGFR-mutated lung cancers. Moreover, decreased expression of the inhibitory $\mathrm{I}_{\kappa} \mathrm{B} \alpha$ protein is associated with resistance to erlotinib and is predictive of worse progression-free survival in patients suffering from lung cancer [49]. Consistent with a role of NF- $\mathrm{KB}$ as a mediator of resistance to EGFR inhibitors, quinacrine overcomes resistance to erlotinib, at least by decreasing the level of SSRP1, an active subunit of the 
facilitates chromatin transcription (FACT) complex that promotes NF- $\mathrm{B}$ transcriptional activity [50].

The crosstalk between EGFR- and NF-кB-activating cascades is particularly relevant in tumor-initiating cells, which crucially promote drug resistance [51,52]. Integrin $\alpha_{\mathrm{v}} \beta_{3}$-expressing tumor-initiating cells from breast, lung, and pancreatic carcinomas are resistant to EGFR inhibitors [53]. NF- $\mathrm{KB}$ activation contributes to this phenotype because integrin $\alpha_{\mathrm{v}} \beta_{3}$ drives tumor stemness and resistance to EGFR inhibitors by interacting with Kirsten rat sarcoma viral oncogene homolog GTPase (KRAS) through galectin-3 to promote the sequential activation of both GTPase v-Ral simian leukemia oncogene homolog B GTPase (RALB) and TANK-binding kinase-1 (TBK1), which then targets c-Rel, a NF-кB protein [53]. Interestingly, this pathway does not require the binding of any ligand to integrin $\alpha_{\mathrm{v}} \beta_{3}$, demonstrating that resistance to EGFR inhibitors can be cell autonomous.

Cell intrinsic mechanisms of resistance to anticancer therapies have also been described in glioblastomas, the most common malignant primary brain cancer of adults. 40-50\% of glioblastomas show EGFR gene amplification and/or mutations, which, in both cases, cause constitutive EGFR signaling [15]. The most common activating EGFR mutation (EGFRvIII) results from a deletion of exons 2-7 of the EGFR gene, which causes an in-frame deletion of 267 amino acids from the receptor in its extracellular domain. As a consequence, EGFRvIII cannot bind to any ligand and is constitutively active [14]. EGFRvIII triggers mTORC2 activation, a complex composed of the kinase mTOR bound to unique regulatory proteins, including Rictor and SIN1 [54]. mTORC2 signals to NF-кB through an AKT-independent pathway to promote proliferation, survival, and cisplatin resistance in glioblastomas [55] (Figure 4). Although it is currently unclear whether this pathway is specifically induced in glioma cells resistant to EGFR inhibitors, this study nevertheless shows that $\mathrm{NF}-\kappa \mathrm{B}$ also acts as a central player in chemotherapy resistance in glioblastoma cells harboring constitutivelyactive EGFR. EGFRvIII also activates NF-кB in glioma cells by assembling a signaling platform with TNF receptor-associated protein 2 (TRAF2), RIPK1, both the cIAP1 and cIAP2 (cellular inhibitor of apoptosis 1/2) E3 ligases, as well as TAK1 [56]. RIPK1 becomes polyubiquitinated in a K63-linked, non-degradative manner to trigger TAK1 and subsequent IKK $\beta$ phosphorylation.

All these pathways occur in a cell autonomous manner, but it is now increasingly obvious that the microenvironment also provides NF- $\mathrm{kB}$-activating signals to promote resistance to EGFR inhibitors. TNF $\alpha$, mainly synthesized by tumor-associated macrophages upon activation of TLR-dependent pathways, acts as a paracrine signal to trigger NF- $\mathrm{B}$ activation in glioma cells [57]. This pathway involves the activation of the serine/threonine kinase

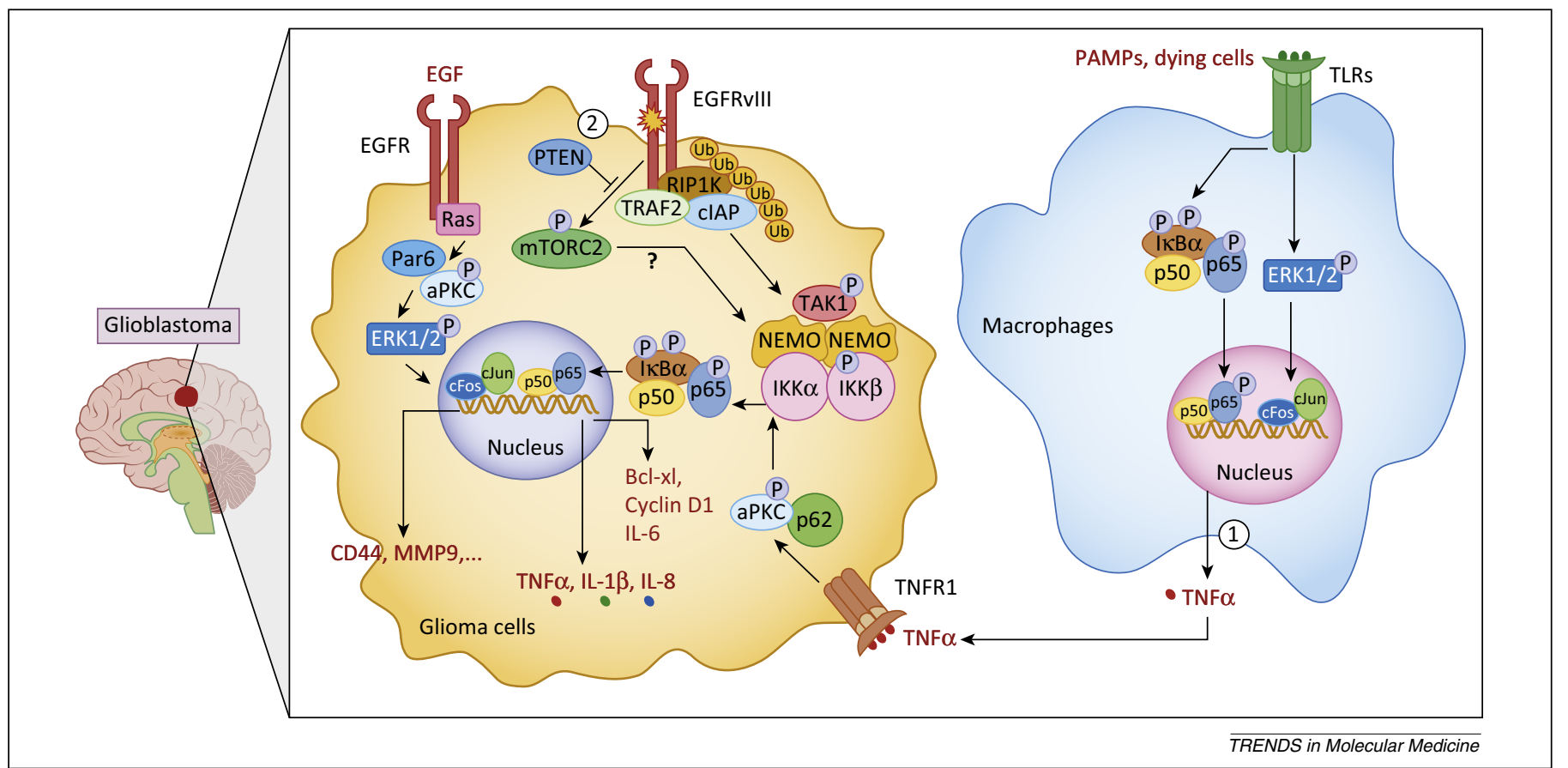

Figure 4. Mechanisms by which NF-kB promotes resistance to chemotherapy in glioblastomas. (1) The production of TNF $\alpha$ by macrophages promotes resistance to EGFR inhibitors through NF-KB activation in glioma cells. Tumor-associated macrophages sense dying cells and pathogen-associated molecular patterns (PAMPs) through TLRs to trigger IKK-dependent NF-KB and ERK1/2 activation. These pathways promote TNF $\alpha$ synthesis, which acts in a paracrine fashion to activate NF- $\mathrm{B}$ through TNFR1dependent aPKC phosphorylation on residues $403 / 410$ in glioma cells [57]. aPKC, whose activation requires the scaffold protein p62, triggers IKK $\beta$ (not illustrated) and IKB $\alpha$ phosphorylation to promote the nuclear translocation of NF-кB subunits $\mathrm{p} 50$ and $\mathrm{p} 65$. This signaling cascade ultimately induces the expression of proinflammatory cytokines (TNF $\alpha, I L-1 \beta, I L-8)$, which establish a positive loop to further activate NF-kB in glioma cells. It is important to note that aPKC activation also occurs upon EGFR

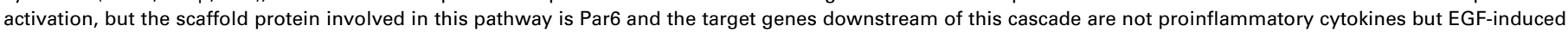
candidates such as CD44 and the matrix metalloproteinase MMP9. Whether, and how, these candidates precisely promote resistance to EGFR inhibitors in glioma cells remains to be clarified. (2) A second pathway underlying resistance to chemotherapy involves an EGFRvIll-dependent (but Akt-independent) signaling pathway that leads to mTORC2 activation in glioma cells. Signaling from the EGFRvIlI mutant triggers mTORC2 phosphorylation, a pathway negatively regulated by PTEN. mTORC2 activation

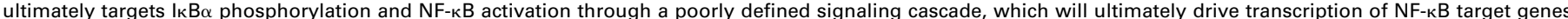
such as Bcl-xl, cyclin D1, and IL-6. EGFRvIII expression also promotes cell survival in glioma cells through K63-linked polyubiquitination of RIPK1 by c-IAPs bound to TRAF2. This signaling complex culminates in NF- $\mathrm{KB}$ activation through TAK1-dependent IKK $\beta$ phosphorylation [56]. 
atypical protein kinase $\mathrm{C}$ (aPKC) bound to the scaffold protein $\mathrm{p} 62$ to induce the expression of proinflammatory cytokines through NF-кB. Interestingly, another pool of aPKC interacting with the scaffold protein Par6 is specifically activated upon EGF stimulation, and drives the expression of another set of genes such as CD44 and MMP9 (Figure 4). Targeting aPKC activity causes tumor regression in EGFR kinase inhibitor-resistant glioblastoma [57].

Combining EGFR and NF-кB inhibitors: the way to go? The demonstration that $\mathrm{NF}-\kappa \mathrm{B}$ contributes to resistance to EGFR inhibitors suggests that the simultaneous inhibition of both EGFR and NF-кB activity might be a therapeutic strategy to circumvent resistance. While tempting, it is currently unclear whether patients would benefit from this combination. Preclinical studies to treat HNSCCs have been conducted to address this issue because both EGFR and NF- $\mathrm{kB}$ activity are enhanced in a high percentage of these malignancies [58]. The EGFR-targeting cetuximab used in combination with radiation prolonged overall survival but failed to reduce the incidence of metastasis, and the response rate with cetuximab administered alone is not above $13 \%$ [59]. One mechanism underlying resistance to cetuximab results from the expression of the EGFRvIII mutant, which has a truncated ligand-binding domain, and signals to Lyn, a member of the SRC family of kinases and to STAT3 in up to $42 \%$ cases of HNSCC [60,61]. Although the EGFR inhibitor gefitinib showed some response in clinical trials of HNSCC by decreasing EGFR, MEK1, and NF-кB p65 phosphorylation, it failed to interfere with these oncogenic pathways in nonresponder patients [62]. Therefore, there was a need to combine EGFR inhibitors with other targeted therapies.

Bortezomib, a proteasome inhibitor, triggers cell apo-

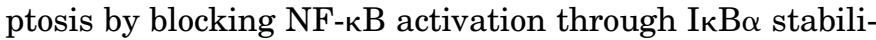
zation [63]. However, bortezomib showed limited clinical efficacy in HNSCC because NF-кB activation through the noncanonical pathway remained unchanged $[64,65]$. Moreover, bortezomib did not impact on other prosurvival pathways driven by STAT3 and ERK1/2 phosphorylation [64]. Therefore, the combination of bortezomib with EGFR inhibitors was expected to improve the clinical response. A Phase I clinical trial was carried out with patients suffering from HNSCC to assess preclinical evidence for combining the EGFR inhibitor cetuximab with bortezomib and radiation therapy [66]. This combination showed unexpectedly early progression due to EGFR stabilization, increased prosurvival signaling, and enhanced cytokine expression. Bortezomib attenuated the cytotoxic effects of cetuximab and radiation by limiting EGFR degradation through the proteasome [66]. Proteasome inhibition actually enhanced MAPK, AKT, and STAT3 prosurvival pathways through both EGFR-dependent and -independent mechanisms [67]. These studies highlighted the need to design new combinatorial approaches by using a morespecific NF-кB inhibitor than bortezomib, which blocks the degradation of $\mathrm{I} \kappa \mathrm{B} \alpha$ as well as of numerous other proteins, including key oncogenic products [68]. Interestingly, both IKK $\alpha$ and IKK $\beta$ are aberrantly activated in HNSCC, act as mediators of NF-KB activation, and enhance EGFR signaling in HNSCC [43]. Consistently, wedelactone, a dual IKK $\alpha / \beta$ inhibitor, more effectively inhibited NF-кB activation than MLN120b, a specific IKK $\beta$ inhibitor $[43,69]$. Moreover, 17-DMAG, a geldanamycin derivative that inhibits the heat shock protein $90 \mathrm{kDa}$ (HSP90), a molecular chaperone involved in both IKK $\alpha / \beta$ - and EGFRdependent signaling cascades, was more effective at triggering cell death than MLN120b alone [43,70]. Therefore, these data demonstrated why specific IKK $\beta$ inhibitors have not led to expected efficacies in preclinical studies, and suggest that combining dual IKK $\alpha / \beta$ and EGFR inhibitors would be more successful, at least for epithelial malignancies.

\section{Does NF-kB play any role in the toxicity of EGFR inhibitors?}

Although NF-кB activation limits the clinical response to EGFR inhibitors, it is currently unclear whether the side effects commonly reported with these drugs would still occur by targeting NF- $\mathrm{KB}$. Cutaneous side effects are often described in patients treated with EGFR inhibitors (monoclonal antibodies or small-molecule inhibitors) [71,72]. They include hair loss, acneiform eruption, paronychia, and xerosis. Mechanistically, these symptoms result from the inflammation-prone response of keratinocytes to early infiltration of macrophages and mast cells into the skin, as well as from an increased percentage of circulating granulocytes and platelets, but decreased percentage of lymphocytes in the plasma [73,74]. The genetic inactivation of EGFR in the epidermis mimics those symptoms, suggesting that EGFR expression maintains skin immune homeostasis [73]. EGFR-deficient keratinocytes overexpress a variety of chemokines and cytokines (such as IL- $1 \beta, \mathrm{TNF} \alpha$, and IL-6) that are known to be regulated by NF-кB-dependent pathways. However, the genetic inactivation of single proinflammatory pathways (e.g., TNFR1/R2, Myd88) did not reverse the induction and maintenance of the skin phenotype in the EGFR-deficient mouse model [73,74]. Therefore, it is unlikely that targeting NF-кB alone will improve cutaneous toxicity seen with EGFR inhibitors.

\section{Concluding remarks and future perspectives}

Recent studies have elucidated the molecular mechanisms by which targeted therapies lead to resistance in epithelial malignancies displaying constitutive signaling from ERBB receptors. The simultaneous administration of specific inhibitors is the most logical approach to treat tumors with intrinsic or acquired resistance. Combining ERBB and $\mathrm{NF}-\kappa \mathrm{B}$ inhibitors is very promising, given the key role of NF- $\kappa \mathrm{B}$ in tumors resistant to EGFR inhibitors; however, key issues remain unresolved (Box 3). New irreversible EGFR inhibitors are currently being tested to treat lung tumors harboring the T790M mutation. Given the genomic plasticity of many aggressive solid tumors, including lung carcinomas, it is likely that new oncogenic mutations and/ or activation of signaling pathways will occur in patients treated with these irreversible EGFR inhibitors. Whether $\mathrm{NF}-\kappa \mathrm{B}$ activation contributes or not to this resistance remains to be clarified. In addition, it is currently unclear which NF-кB inhibitor should be used in combination with ERBB inhibitors for the best clinical response. A variety of 


\section{Box 3. Outstanding questions}

- What is the best combination of EGFR and NF-кB inhibitors to use in the clinic?

- Does NF-кB promote resistance in solid tumors harboring the T790M mutation?

- Does the simultaneous inhibition of HER2 and HER3 lead to resistance, and, if so, does NF-kB play a role?

- Does the simultaneous inhibition of both NF-kB and EGFRdependent signaling pathways in patients improve cutaneous side effects typically seen with EGFR inhibitors?

- The ligand-induced versus constitutive EGFR-dependent signaling may rely on partially distinct signaling complexes, but how can we translate these findings to the clinic?

IKK inhibitors have been developed over the past 15 years, and some have been used in clinical trials [75-77]. However, none of them have been approved so far, at least because some doubts on the interest of targeting IKK $\beta$ itself were raised [77]. As recent studies carried out in HNSCC indicated that specific IKK $\beta$ inhibitors do not totally block NF$\kappa \mathrm{B}$ activation, it is likely that other NF-кB inhibitors will need to be tested in combination with ERBB inhibitors in all epithelial malignancies showing constitutive signaling from ERBB receptors [43]. Being over-specific (i.e., by targeting only IKK $\beta$ ) may not be the best strategy. Finally, combining EGFR or HER2 inhibitors with HER3 blocking antibodies to circumvent resistance hold promise for the future, but it is also likely that some cancer cells will ultimately escape from this therapeutic strategy. If so, the potential role of $\mathrm{NF}-\kappa \mathrm{B}$ in these resistant cells will need to be explored.

\section{Acknowledgments}

Our laboratory is supported by grants from the Fonds National de la Recherche Scientifique (FNRS), TELEVIE, the University of Liege [Concerted Research Action Program (BIO-ACET) and Fonds Spéciaux (C-11/03)], the Centre Anti-Cancéreux, the Leon Fredericq Fondation (University of Liege), as well as by WELBIO. A.C. is Senior Research Associate at the FNRS

\section{References}

1 Sordella, R. et al. (2004) Gefitinib-sensitizing EGFR mutations in lung cancer activate anti-apoptotic pathways. Science 305, 1163-1167

2 Yarden, Y. and Sliwkowski, M.X. (2001) Untangling the ErbB signalling network. Nat. Rev. Mol. Cell Biol. 2, 127-137

3 Shoelson, S.E. (1997) SH2 and PTB domain interactions in tyrosine kinase signal transduction. Curr. Opin. Chem. Biol. 1, 227-234

4 Morandell, S. et al. (2008) Quantitative proteomics and phosphoproteomics reveal novel insights into complexity and dynamics of the EGFR signaling network. Proteomics 8, 4383-4401

5 Lowenstein, E.J. et al. (1992) The SH2 and SH3 domain-containing protein GRB2 links receptor tyrosine kinases to ras signaling. Cell 70, 431-442

6 Okutani, T. et al. (1994) Grb2/Ash binds directly to tyrosines 1068 and 1086 and indirectly to tyrosine 1148 of activated human epidermal growth factor receptors in intact cells. J. Biol. Chem. 269, 31310-31314

7 Sakaguchi, K. et al. (1998) She phosphotyrosine-binding domain dominantly interacts with epidermal growth factor receptors and mediates Ras activation in intact cells. Mol. Endocrinol. 12, 536-543

8 Olayioye, M.A. et al. (2000) The ErbB signaling network: receptor heterodimerization in development and cancer. EMBO J. 19, 3159-3167

9 Wykosky, J. et al. (2011) Therapeutic targeting of epidermal growth factor receptor in human cancer: successes and limitations. Chin. J. Cancer 30, 5-12

10 Baselga, J. and Swain, S.M. (2009) Novel anticancer targets: revisiting ERBB2 and discovering ERBB3. Nat. Rev. Cancer 9, 463-475
11 Kris, M.G. et al. (2003) Efficacy of gefitinib, an inhibitor of the epidermal growth factor receptor tyrosine kinase, in symptomatic patients with non-small cell lung cancer: a randomized trial. JAMA 290, 2149-2158

12 Lynch, T.J. et al. (2004) Activating mutations in the epidermal growth factor receptor underlying responsiveness of non-small-cell lung cancer to gefitinib. N. Engl. J. Med. 350, 2129-2139

13 Paez, J.G. et al. (2004) EGFR mutations in lung cancer: correlation with clinical response to gefitinib therapy. Science 304, 1497-1500

14 Ekstrand, A.J. et al. (1991) Genes for epidermal growth factor receptor, transforming growth factor alpha, and epidermal growth factor and their expression in human gliomas in vivo. Cancer Res. 51, 2164-2172

15 Wong, A.J. et al. (1992) Structural alterations of the epidermal growth factor receptor gene in human gliomas. Proc. Natl. Acad. Sci. U.S.A. 89, 2965-2969

16 Sharma, S.V. et al. (2007) Epidermal growth factor receptor mutations in lung cancer. Nat. Rev. Cancer 7, 169-181

17 Cheng, L. et al. (2012) Molecular pathology of lung cancer: key to personalized medicine. Mod. Pathol. 25, 347-369

18 Kobayashi, S. et al. (2005) EGFR mutation and resistance of non-smallcell lung cancer to gefitinib. N. Engl. J. Med. 352, 786-792

$19 \mathrm{Yu}$, H.A. et al. (2013) Analysis of tumor specimens at the time of acquired resistance to EGFR-TKI therapy in 155 patients with EGFR-mutant lung cancers. Clin. Cancer Res. 19, 2240-2247

20 Imai, K. and Takaoka, A. (2006) Comparing antibody and smallmolecule therapies for cancer. Nat. Rev. Cancer 6, 714-727

21 Zhou, W. et al. (2009) Novel mutant-selective EGFR kinase inhibitors against EGFR T790M. Nature 462, 1070-1074

22 Quesnelle, K.M. and Grandis, J.R. (2011) Dual kinase inhibition of EGFR and HER2 overcomes resistance to cetuximab in a novel in vivo model of acquired cetuximab resistance. Clin. Cancer Res. 17, $5935-5944$

23 Engelman, J.A. et al. (2007) MET amplification leads to gefitinib resistance in lung cancer by activating ERBB3 signaling. Science 316, 1039-1043

24 Sergina, N.V. et al. (2007) Escape from HER-family tyrosine kinase inhibitor therapy by the kinase-inactive HER3. Nature 445, 437-441

25 Wheeler, D.L. et al. (2008) Mechanisms of acquired resistance to cetuximab: role of HER (ErbB) family members. Oncogene 27, 3944-3956

26 Pinkas-Kramarski, R. et al. (1996) Diversification of Neu differentiation factor and epidermal growth factor signaling by combinatorial receptor interactions. EMBO J. 15, 2452-2467

27 Campbell, M.R. et al. (2010) HER3 comes of age: new insights into its functions and role in signaling, tumor biology, and cancer therapy. Clin. Cancer Res. 16, 1373-1383

$28 \mathrm{Ma}$, J. et al. (2014) Targeting of erbB3 receptor to overcome resistance in cancer treatment. Mol. Cancer 13, 105

29 Ghosh, S. and Hayden, M.S. (2008) New regulators of NF-kappaB in inflammation. Nat. Rev. Immunol. 8, 837-848

30 Biswas, D.K. et al. (2000) Epidermal growth factor-induced nuclear factor kappa B activation: A major pathway of cell-cycle progression in estrogen-receptor negative breast cancer cells. Proc. Natl. Acad. Sci. U.S.A. 97, 8542-8547

31 Sun, L. and Carpenter, G. (1998) Epidermal growth factor activation of NF-kappaB is mediated through IkappaBalpha degradation and intracellular free calcium. Oncogene 16, 2095-2102

32 Biswas, D.K. et al. (2004) NF-kappa B activation in human breast cancer specimens and its role in cell proliferation and apoptosis. Proc. Natl. Acad. Sci. U.S.A. 101, 10137-10142

33 Le Page, C. et al. (2005) EGFR and Her-2 regulate the constitutive activation of NF-kappaB in PC-3 prostate cancer cells. Prostate 65, 130-140

34 Yang, W. et al. (2012) EGFR-induced and PKCepsilon monoubiquitylation-dependent NF-kappaB activation upregulates PKM2 expression and promotes tumorigenesis. Mol. Cell 48, 771-784

35 Jiang, T. et al. (2011) CARMA3 is crucial for EGFR-Induced activation of NF-kappaB and tumor progression. Cancer Res. 71, 2183-2192

36 McAllister-Lucas, L.M. et al. (2007) CARMA3/Bcl10/MALT1 dependent NF-kappaB activation mediates angiotensin II-responsive inflammatory signaling in nonimmune cells. Proc. Natl. Acad. Sci. U.S.A. 104, 139-144 
37 Mahanivong, C. et al. (2008) Protein kinase C alpha-CARMA3 signaling axis links Ras to NF-kappa B for lysophosphatidic acidinduced urokinase plasminogen activator expression in ovarian cancer cells. Oncogene 27, 1273-1280

38 Thome, M. (2008) Multifunctional roles for MALT1 in T-cell activation. Nat. Rev. Immunol. 8, 495-500

39 De, S. et al. (2014) EGF receptor uses SOS1 to drive constitutive activation of NFkappaB in cancer cells. Proc. Natl. Acad. Sci. U.S.A. $111,11721-11726$

40 Zhao, C. et al. (2007) Phospholipase D2-generated phosphatidic acid couples EGFR stimulation to Ras activation by Sos. Nat. Cell Biol. 9, 706-712

41 Shostak, K. et al. (2014) NF-kappaB-induced KIAA1199 promotes survival through EGFR signalling. Nat. Commun. 5, 5232

42 Kuscu, C. et al. (2012) Transcriptional and epigenetic regulation of KIAA1199 gene expression in human breast cancer. PLoS ONE 7, e44661

43 Nottingham, L.K. et al. (2014) Aberrant IKKalpha and IKKbeta cooperatively activate NF-kappaB and induce EGFR/AP1 signaling to promote survival and migration of head and neck cancer. Oncogene $33,1135-1147$

44 Ramnarain, D.B. et al. (2008) RIP1 links inflammatory and growth factor signaling pathways by regulating expression of the EGFR. Cell Death Differ. 15, 344-353

45 Bentires-Alj, M. et al. (2003) NF-kappaB transcription factor induces drug resistance through MDR1 expression in cancer cells. Oncogene 22, 90-97

46 Guo, C. and Stark, G.R. (2011) FER tyrosine kinase (FER) overexpression mediates resistance to quinacrine through EGFdependent activation of NF-kappaB. Proc. Natl. Acad. Sci. U.S.A. 108, 7968-7973

$47 \mathrm{Kim}$, L. and Wong, T.W. (1998) Growth factor-dependent phosphorylation of the actin-binding protein cortactin is mediated by the cytoplasmic tyrosine kinase FER. J. Biol. Chem. 273, 23542-23548

48 Kim, L. and Wong, T.W. (1995) The cytoplasmic tyrosine kinase FER is associated with the catenin-like substrate pp120 and is activated by growth factors. Mol. Cell. Biol. 15, 4553-4561

49 Bivona, T.G. et al. (2011) FAS and NF-kappaB signalling modulate dependence of lung cancers on mutant EGFR. Nature 471, 523-526

50 Dermawan, J.K. et al. (2014) Quinacrine overcomes resistance to erlotinib by inhibiting FACT, NF-kappaB, and cell-cycle progression in non-small cell lung cancer. Mol. Cancer Ther. 13, 2203-2214

51 Dean, M. et al. (2005) Tumour stem cells and drug resistance. Nat. Rev. Cancer 5, 275-284

52 Singh, A. and Settleman, J. (2010) EMT, cancer stem cells and drug resistance: an emerging axis of evil in the war on cancer. Oncogene 29, $4741-4751$

53 Seguin, L. et al. (2014) An integrin beta(3)-KRAS-RalB complex drives tumour stemness and resistance to EGFR inhibition. Nat. Cell Biol. 16 , $457-468$

54 Guertin, D.A. and Sabatini, D.M. (2009) The pharmacology of mTOR inhibition. Sci. Signal. 2, pe24

55 Tanaka, K. et al. (2011) Oncogenic EGFR signaling activates an mTORC2-NF-kappaB pathway that promotes chemotherapy resistance. Cancer Discov. 1, 524-538

56 Puliyappadamba, V.T. et al. (2013) Opposing effect of EGFRWT on EGFRvIII-mediated NF-kappaB activation with RIP1 as a cell death switch. Cell Rep. 4, 764-775

57 Kusne, Y. et al. (2014) Targeting aPKC disables oncogenic signaling by both the EGFR and the proinflammatory cytokine TNFalpha in glioblastoma. Sci. Signal. 7, ra75

58 Van Waes, C. (2007) Nuclear factor-kappaB in development, prevention, and therapy of cancer. Clin. Cancer Res. 13, 1076-1082

59 Vermorken, J.B. et al. (2007) Open-label, uncontrolled, multicenter phase II study to evaluate the efficacy and toxicity of cetuximab as a single agent in patients with recurrent and/or metastatic squamous cell carcinoma of the head and neck who failed to respond to platinumbased therapy. J. Clin. Oncol. 25, 2171-2177

60 Wheeler, S.E. et al. (2010) Epidermal growth factor receptor variant III mediates head and neck cancer cell invasion via STAT3 activation. Oncogene 29, 5135-5145

61 Wheeler, S.E. et al. (2012) Lyn kinase mediates cell motility and tumor growth in EGFRvIII-expressing head and neck cancer. Clin. Cancer Res. 18, 2850-2860

62 Pernas, F.G. et al. (2009) Proteomic signatures of epidermal growth factor receptor and survival signal pathways correspond to gefitinib sensitivity in head and neck cancer. Clin. Cancer Res. 15, 2361-2372

63 Adams, J. and Kauffman, M. (2004) Development of the proteasome inhibitor Velcade (bortezomib). Cancer Invest. 22, 304-311

64 Allen, C. et al. (2008) Bortezomib-induced apoptosis with limited clinical response is accompanied by inhibition of canonical but not alternative nuclear factor-\{kappa\}B subunits in head and neck cancer. Clin. Cancer Res. 14, 4175-4185

65 Allen, C.T. et al. (2015) CCR 20th Anniversary Commentary: preclinical study of proteasome inhibitor bortezomib in head and neck cancer. Clin. Cancer Res. 21, 942-943

66 Argiris, A. et al. (2011) Early tumor progression associated with enhanced EGFR signaling with bortezomib, cetuximab, and radiotherapy for head and neck cancer. Clin. Cancer Res. 17, 5755-5764

67 Sloss, C.M. et al. (2008) Proteasome inhibition activates epidermal growth factor receptor (EGFR) and EGFR-independent mitogenic kinase signaling pathways in pancreatic cancer cells. Clin. Cancer Res. 14, 5116-5123

68 Skaar, J.R. et al. (2014) SCF ubiquitin ligase-targeted therapies. Nat. Rev. Drug Discov. 13, 889-903

69 Kobori, M. et al. (2004) Wedelolactone suppresses LPS-induced caspase-11 expression by directly inhibiting the IKK complex. Cell Death Differ. 11, 123-130

70 Hertlein, E. et al. (2010) 17-DMAG targets the nuclear factor-kappaB family of proteins to induce apoptosis in chronic lymphocytic leukemia: clinical implications of HSP90 inhibition. Blood 116, 45-53

$71 \mathrm{Hu}$, J.C. et al. (2007) Cutaneous side effects of epidermal growth factor receptor inhibitors: clinical presentation, pathogenesis, and management. J. Am. Acad. Dermatol. 56, 317-326

72 Lacouture, M.E. (2006) Mechanisms of cutaneous toxicities to EGFR inhibitors. Nat. Rev. Cancer 6, 803-812

73 Mascia, F. et al. (2013) Genetic ablation of epidermal EGFR reveals the dynamic origin of adverse effects of anti-EGFR therapy. Sci. Transl. Med. 5, 199ra110

74 Lichtenberger, B.M. et al. (2013) Epidermal EGFR controls cutaneous host defense and prevents inflammation. Sci. Transl. Med. 5, 199ra111

75 Lee, D.F. and Hung, M.C. (2008) Advances in targeting IKK and IKK-related kinases for cancer therapy. Clin. Cancer Res. 14, $5656-5662$

76 Karin, M. et al. (2004) The IKK NF-kappa B system: a treasure trove for drug development. Nat. Rev. Drug Discov. 3, 17-26

77 Verstrepen, L. and Beyaert, R. (2014) Receptor proximal kinases in NF-kappaB signaling as potential therapeutic targets in cancer and inflammation. Biochem. Pharmacol. 92, 519-529

78 Popovic, D. et al. (2014) Ubiquitination in disease pathogenesis and treatment. Nat. Med. 20, 1242-1253

79 Hynes, N.E. and MacDonald, G. (2009) ErbB receptors and signaling pathways in cancer. Curr. Opin. Cell Biol. 21, 177-184

80 Roskoski, R., Jr (2014) The ErbB/HER family of protein-tyrosine kinases and cancer. Pharmacol. Res. 79, 34-74

81 Hayden, M.S. and Ghosh, S. (2012) NF-kappaB, the first quartercentury: remarkable progress and outstanding questions. Genes Dev. 26, 203-234

82 Chen, J. and Chen, Z.J. (2013) Regulation of NF-kappaB by ubiquitination. Curr. Opin. Immunol. 25, 4-12 